\title{
Maximally non-Markovian quantum dynamics without environment backflow of information
}

\author{
Adrián A. Budini \\ Consejo Nacional de Investigaciones Científicas y Técnicas (CONICET), \\ Centro Atómico Bariloche, Avenida E. Bustillo Km 9.5, (8400) Bariloche, \\ Argentina, and Universidad Tecnológica Nacional (UTN-FRBA), \\ Fanny Newbery 111, (8400) Bariloche, Argentina
}

(Dated: April 11, 2018)

\begin{abstract}
The degree of non-Markovianity allows to characterizing quantum evolutions that depart from a Markovian regime in a similar way as Schmidt number measures the degree of entanglement of pure states. Maximally non-Markovian dynamics are the analogous of maximally entangled states [D. Chruściński and S. Maniscalco, Phys. Rev. Lett. 112, 120404 (2014)]. Here, we demonstrate that there exists a class of maximally non-Markovian quantum evolutions where the associated environment (degrees of freedom not belonging to the system) obeys a Markovian (memoryless) dynamics, which in turn is unperturbed by the system state or dynamics. These properties imply the absence of any "physical environment-to-system backflow of information." Non-Markovian features (as usual in quantum systems coupled to dissipative classical degrees of freedom) arise from a unidirectional dependence of the system dynamics on the reservoir states.
\end{abstract}

\section{INTRODUCTION}

In contrast to classical stochastic systems, for open quantum systems [1] the definition of memory, or equivalently departure from a memoryless (Markovian) regime [1, 2], is much more subtle. The theory of quantum dynamical semigroups 3] gives a rigorous basis for defining Markovian dynamics, where the time evolution of the system density matrix is given by the so called Lindblad equations (or Gorini-Kossakowski-SudarshanLindblad equations). Assuming that all available information is encoded in the system density matrix, any departure in the properties of its propagator with respect to that of a quantum dynamical semigroup may be utilized as an indicator of the presence of memory, or equivalently, for defining a quantum non-Markovian regime [4, 5].

The previous point of view was introduced in the seminal contribution of Breuer, Laine, and Piilo [6], where the memory indicator is given by the (non-monotonous) behavior of the distinguishability between two initial states. Since then, many other indicators and measures were introduced, such as for example based on the divisibility of the propagator [7 10$]$, geometry of the set of accessible states [11], negativity of the dissipative rates in a canonical form of the quantum master equation [12], nonMarkovianity degree [13, 14], spectra of the dynamical map [15], quantum regression theorem [16], power spectrum [17], Fisher information flow [18], mutual information [19], fidelity 20], and accessible information [21] just to name a few [4, 5]. In addition, many aspects were analyzed such as for example the relation with the definition of non-Markovian classical stochastic processes [22, 23], and the correspondence between the different (inequivalent) non-Markovian indicators and measures [24 29].

The authors of Ref. [6] also introduced a new perspective for understanding non-Markovian or memory effects: one can read any departure from a Markovian regime (measured, in general, with any of the previous indica- tors) in terms of a backflow of information from the reservoir to the system. Instead, the memoryless (Markovian) case corresponds to a unidirectional loss of information from the system to the reservoir. This conceptual frame was criticized in Ref. [30]. On the other hand, there are physical situations where it has a clear meaning. For example, in a Markovian regime, an excitation of a quantum optical transition is transferred unidirectionally from the system to the environment [1]. In the other extreme, for a two-level system interacting with a quantized harmonic oscillator (Jaynes-Cumming model [1]) an excitation is continuously transferred back and forth between the (two-level) system and the environment (quantum harmonic oscillator). Spin-boson models [1] interpolate between both extreme behaviors. This kind of bidirectional physical backflow of information, which is mediated between the system and the environment by a physical variable such as energy or heat, was analyzed in Refs. [31 33] and recently studied experimentally in Ref. [34].

It is important to notice that memory effects (as detected by any of the proposed non-Markovian indicators based on the system dynamics) may happen without a "physical environment-to-system backflow of information." For example, the environment, defined by all degrees of freedom not belonging to the system, may has its own Markovian (time-memoryless) dynamics which in turn is unperturbed by the system dynamics or state. Hence, even when the system dynamics is nonMarkovian, the environment (its partial dynamics) is completely unaware of its coupling with the system.

A class of dynamics with the previous property is given by quantum systems coupled to environments modeled through stochastic Hamiltonians whose fluctuations are written in terms of classical non-white (Gaussian) noises. Even when the system may develops strong nonMarkovian features [35], the environment (the noise) is unaffected by the system. In addition, quantum systems coupled to dissipative classical degrees of freedom 36. 
may lead to the same situation, that is, the system dynamics is non-Markovian while the environment follows a Markovian evolution that is unaffected by the system state. For example, in Ref. [37] a class of time-convoluted non-Markovian master equations were derived over the previous basis, where the non-Markovian indicator is the relative entropy with respect to the stationary state. In addition, a class of non-Markovian collisional models 38] can be recovered in a similar way, where a quantum ancilla system (part of the environment) in a bipartite embedding follows its own independent quantum Markovian Lindblad evolution [39]. More recently, master equations leading to eternal non-Markovianity, a Lindbladlike equation with a time-dependent rate that is negative at all times [12], were derived on similar grounds [40]. The underlying (Markovian) environment dynamics can be read in alternative ways such as random dephasing channels and Markov chains [36].

The main goal of this paper is to extend the previous results [37, 39, 40] to the case of maximally nonMarkovian quantum evolutions. This kind of dynamics was introduced by Chruściński and Maniscalco in Ref. [13]. Studying the positivity of the propagator between two arbitrary times in an extended Hilbert space (divisibility), the authors defined a non-Markovianity degree. This parameter is the analog of Schmidt number in entanglement theory, and allows to compare (to rank) different non-Markovian evolutions. Maximally nonMarkovian quantum evolutions are the analog of maximally entangled states. Here, we demonstrate that there exists of class of such kind of extreme non-Markovian dynamics that are induced by environments whose own dynamics is unperturbed by the system state, that is, without existing a physical environment-to-system backflow of information. The evolutions rely on simple quantumclassical hybrid arranges (collisional models [38] with a finite number of events), where the classical degrees of freedom (the environment) follow their own local in time Markovian evolution 36. Similarly to the results of Refs. [37, 39, 40], non-Markovian effects arise due to a unidirectional dependence of the system evolution on the environment states.

The paper is organized as follows. In Sec. II, different examples of maximally non-Markovian evolutions are introduced. In Sec. III, the examples are derived from hybrid quantum-classical dynamics. In Sec. IV, the more general underlying quantum-classical dynamics that lead to non-Markovian effects without a physical environment backflow of information are characterized. Sec. V is devoted to the Conclusions. Information that supports the main obtained results is provided in the Appendixes.

\section{MAXIMALLY NON-MARKOVIAN EVOLUTIONS}

Maximally non-Markovian evolutions saturate the degree of non-Markovianity. Explicit examples that satisfy the required conditions found in [13] are provided. The underlying formalism is briefly reviewed in Appendix A. Both dephasing and random unitary maps are given.

\section{A. Dephasing channel}

Consider a qubit system whose density matrix $\rho_{t}$ follows the evolution

$$
\frac{d \rho_{t}}{d t}=\frac{1}{2} \gamma(t)\left(\sigma_{z} \rho_{t} \sigma_{z}-\rho_{t}\right)
$$

where $\gamma(t)$ is a time-dependent rate and $\sigma_{z}$ is the $z$-Pauli matrix. The solution can be written as

$$
\rho_{t}=\left(\begin{array}{cc}
\rho_{11} & \rho_{12} e^{-\Gamma(t)} \\
\rho_{21} e^{-\Gamma(t)} & \rho_{22}
\end{array}\right),
$$

where $\Gamma(t) \equiv \int_{0}^{t} \gamma(\tau) d \tau$. Eq. (2) shows the dephasing property of the solution map, that is, only coherences are affected.

The evolution is completely positive if and only if $\Gamma(t) \geq 0$. Furthermore, the evolution is maximally nonMarkovian if [13]

$$
\lim _{t \rightarrow \infty} \Gamma(t)=\lim _{t \rightarrow \infty} \int_{0}^{t} \gamma(\tau) d \tau=0 .
$$

This simple condition implies the extreme nonMarkovian property $\lim _{t \rightarrow \infty} \rho_{t}=\rho_{0}$ [see Eq. (2)]. Hence, in the long time limit (stationary state) the initial density matrix is recovered.

In order to fulfill the previous condition, we propose the following time dependent rate

$$
\gamma(t)=2 \gamma \frac{1-\gamma t}{e^{\gamma t}-2 \gamma t},
$$

where the free scaling parameter $\gamma>0$ determines the initial rate value, $\lim _{t \rightarrow 0} \gamma(t)=2 \gamma$. Its time integral is given by

$$
\Gamma(t)=\ln \left[\frac{1}{1-2 \gamma t e^{-\gamma t}}\right], \quad \lim _{t \rightarrow \infty} \Gamma(t)=0,
$$

which is positive, $\Gamma(t) \geq 0$, and also satisfies condition (3). Hence, the corresponding evolution [Eq. (1)] is completely positive and maximally non-Markovian. In Fig. 1 we plot both the rate $\gamma(t)$ and its time integral $\Gamma(t)$. The time dependent rate is a smooth function that does not present any divergence and consistently assumes positive and negative values.

The coherences behavior in Eq. (2) follows straightforwardly as $e^{-\Gamma(t)}=\left[1-2 \gamma t e^{-\gamma t}\right]$. From this solution, it is possible to rewriting the evolution (11) with a timeconvoluted structure,

$$
\frac{d \rho_{t}}{d t}=\frac{1}{2} \int_{0}^{t} d t^{\prime} k\left(t-t^{\prime}\right)\left(\sigma_{z} \rho_{t^{\prime}} \sigma_{z}-\rho_{t^{\prime}}\right)
$$




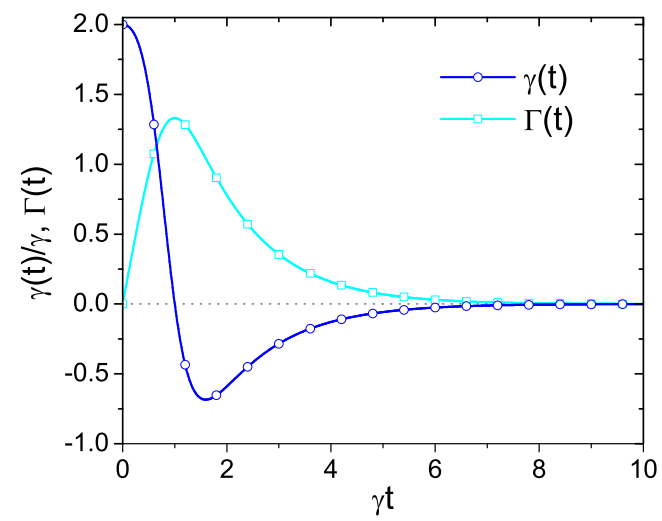

FIG. 1: Time dependent rate $\gamma(t)$ and its integral $\Gamma(t)$, Eqs. (4) and (5) respectively, as a function of time for the maximally non-Markovian dephasing dynamics defined by Eq. (11).

with memory kernel

$$
k(t)=2 \gamma[\delta(t)-\gamma \sin (\gamma t)],
$$

where $\delta(t)$ is the delta-Dirac function. This kind of kernel, with both local and nonlocal in time contributions, were also found in Refs. [37, 40].

\section{B. Random unitary dynamics}

In this case, the qubit dynamics is governed by the time-dependent generator ( $\sigma_{k}$ is the $k$-Pauli matrix)

$$
\frac{d \rho_{t}}{d t}=\frac{1}{2} \sum_{k=1}^{3} \gamma_{k}(t)\left(\sigma_{k} \rho_{t} \sigma_{k}-\rho_{t}\right),
$$

where two decoherence channels were added. The solution is written as a random unitary map,

$$
\rho_{t}=\sum_{\alpha=0}^{3} p_{\alpha}(t) \sigma_{\alpha} \rho_{0} \sigma_{\alpha},
$$

where $\sigma_{0}=\mathbb{I}, 0 \leq p_{\alpha}(t) \leq 1, \sum_{\alpha=0}^{3} p_{\alpha}(t)=1$. The positivity of the weights $\left\{p_{\alpha}(t)\right\}$ guarantee the completely positive condition of the solution map.

The set of probabilities $\left\{p_{\alpha}(t)\right\}$ and the set of time dependent rates $\left\{\gamma_{k}(t)\right\}$ depend each of the other. Given the probabilities, the rates can be expressed as [41]

$$
\gamma_{\alpha}(t)=\frac{1}{2} \sum_{\alpha=0}^{3} H_{\alpha \beta} \frac{d}{d t}\left\{\ln \left[\sum_{\gamma=0}^{3} H_{\beta \gamma} p_{\gamma}(t)\right]\right\},
$$

which implies the relation $\gamma_{0}(t)=-\sum_{k=1}^{3} \gamma_{k}(t)$. The coefficients $\left\{H_{\alpha \beta}\right\}$ correspond to a square four dimensional Hadamard matrix [41]. On the other hand, in Appendix B we find a time-convoluted master equation that is equivalent to Eq. (8) (see also Ref. [42]).

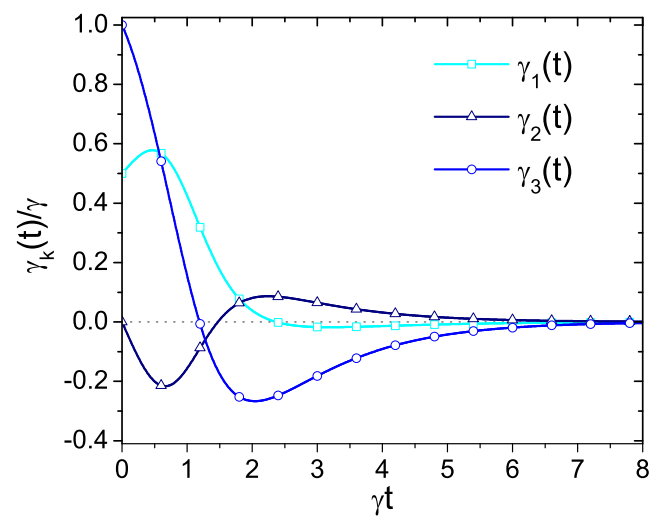

FIG. 2: Time dependent rates $\left\{\gamma_{k}(t)\right\}$ [Eq. (13)] for the maximally non-Markovian random unitary evolution (8) corresponding to the map (9) with probabilities (12).

Defining $\Gamma_{k}(t)=\int_{0}^{t} \gamma_{k}(\tau) d \tau$, the solution map (9) is maximally non-Markovian, for example, if $\gamma_{2}(t)+\gamma_{3}(t) \ngtr$ 0 and [13]

$$
\lim _{t \rightarrow \infty} \Gamma_{1}(t) \geq 0, \quad \lim _{t \rightarrow \infty} \Gamma_{2}(t)=\lim _{t \rightarrow \infty} \Gamma_{3}(t)=0 .
$$

As an explicit example we take the positive and normalized probabilities

$$
\begin{aligned}
& p_{0}(t)=\frac{3}{4}+\frac{1}{4} e^{-\gamma t}(1-2 \gamma t), \\
& p_{1}(t)=\frac{1}{4}\left(1-e^{-\gamma t}\right), \quad p_{2}(t)=0, \\
& p_{3}(t)=\frac{1}{2} \gamma t e^{-\gamma t},
\end{aligned}
$$

where $\gamma>0$. From Eq. (10), the time dependent rates can be written as

$$
\begin{aligned}
\gamma_{1}(t) & =\frac{1}{2} \gamma\left[-g_{a}(t)+g_{b}(t)+g_{c}(t)\right], \\
\gamma_{2}(t) & =\frac{1}{2} \gamma\left[g_{a}(t)-g_{b}(t)+g_{c}(t)\right], \\
\gamma_{3}(t) & =\frac{1}{2} \gamma\left[g_{a}(t)+g_{b}(t)-g_{c}(t)\right],
\end{aligned}
$$

where the auxiliary functions are

$$
\begin{aligned}
g_{a}(t) & \equiv \frac{1-\gamma t}{e^{\gamma t}-\gamma t} \\
g_{b}(t) & \equiv \frac{3-2 \gamma t}{1+e^{\gamma t}-2 \gamma t}, \\
g_{c}(t) & \equiv \frac{1}{1+e^{\gamma t}} .
\end{aligned}
$$

In Fig. 2 we plot the set $\left\{\gamma_{k}(t)\right\}$. All functions are smooth, do not diverge, assume positive and negative values, and vanish asymptotically.

From the expressions for the rates [Eq. (13)] it is possible to obtain $\lim _{t \rightarrow \infty} \Gamma_{1}(t)=\ln (2)$, while $\lim _{t \rightarrow \infty} \Gamma_{2}(t)=$ $\lim _{t \rightarrow \infty} \Gamma_{3}(t)=0$. Furthermore, $\gamma_{2}(t)+\gamma_{3}(t)=\gamma g_{a}(t) \nsupseteq$ 0 . Hence, from Eq. (11) we conclude that the evolution is maximally non-Markovian. 


\section{QUANTUM-CLASSICAL REPRESENTATION OF DYNAMICS}

In the previous section, two maximally non-Markovian evolutions were explicitly defined. Here, we demonstrate that both examples can be recovered from simple quantum-classical dynamics, which admit different representations such as in terms of Markov chains, Lindblad rate equations, and bipartite evolutions. In all cases, the environment follows its own unaffected Markovian dynamics. These examples give a basis for constructing a full class of dynamics with the same memory properties.

\section{A. Dephasing channel}

The dephasing channel is defined by Eq. (1) with timedependent rate (4).

\section{Markov chain representation}

We introduce a classical stochastic system endowed with three different states (Fig. 3). Their probabilities $\left\{P_{i}(t)\right\}, i=0,1,2$, obey by the classical master equation

$$
\begin{aligned}
& \frac{d P_{0}(t)}{d t}=-\gamma P_{0}(t) \\
& \frac{d P_{1}(t)}{d t}=-\gamma P_{1}(t)+\gamma P_{0}(t), \\
& \frac{d P_{2}(t)}{d t}=+\gamma P_{1}(t)
\end{aligned}
$$

with initial condition $P_{0}(0)=1$. Thus, at random times the classical system undergoes the successive transitions $0 \rightarrow 1 \rightarrow 2$. In addition, in each transition the quantum system suffers the disruptive transformation

$$
\rho \rightarrow \sigma_{z} \rho \sigma_{z}
$$

Therefore, the density matrix of the quantum system is

$$
\rho_{t}=P_{0}(t) \rho_{0}+P_{1}(t) \sigma_{z} \rho_{0} \sigma_{z}+P_{2}(t) \sigma_{z}^{2} \rho_{0} \sigma_{z}^{2} .
$$

This solution can be read as a quantum collisional model [38] with a finite number of events.

Eq. (19) can trivially be written with the structure given by Eq. (9), with $p_{0}(t)=P_{0}(t)+P_{2}(t), p_{1}(t)=$ $p_{2}(t)=0$, and $p_{3}(t)=P_{1}(t)$. By solving Eq. (17) with $P_{0}(0)=1$, we get

$$
p_{0}(t)=1-\gamma t \exp (-\gamma t), \quad p_{3}(t)=\gamma t \exp (-\gamma t) .
$$

These expressions in turn, from Eq. (10), lead to the time dependent rate (4), which recovers in consequence the maximally non-Markovian quantum dephasing evolution introduced previously. This result can alternatively be derived from Eq. (19) by obtaining the coherences time behavior.

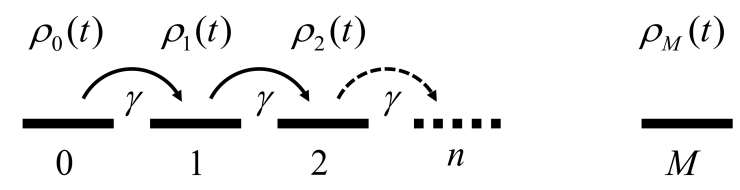

FIG. 3: Graphical representation of the quantum-classical hybrid dynamics. For the dephasing channel [Eqs. (1) and (4)], the environment consists in three (unidirectionally) coupled states, which obey the classical master equation (17). In each transition, the transformation (18) is applied. The quantum state follows from the Lindblad rate evolution (22). Extra coupled states (dashed line) may lead to decoherence and recoherence, Eq. (25). A single uncoupled state $M$ completes the scheme for the random unitary evolution [Eqs. (8) and (13)], whose solution is the mixed state (28).

In the previous stochastic representation, the property $\lim _{t \rightarrow \infty} \rho_{t}=\rho_{0}$ can be understood straightforwardly from the occurrence of two successive flips in the sign of the coherences, which are induced by the transformation $\rho \rightarrow \sigma_{z} \rho \sigma_{z}$. Furthermore, the environment, represented here by the classical system, is completely unaware of the (unidirectional) dependence of the system dynamics on its states.

\section{Lindblad rate equation}

As is well known, the class of stochastic dynamics described previously can be formulated in terms of Lindblad rate equations [36]. These generalized Lindblad equations give the more general evolution for a quantumclassical (hybrid) arrange where the behavior of the classical part is inherently irreversible in time.

The system density matrix is written as

$$
\rho_{t}=\sum_{i=0}^{2} \rho_{i}(t),
$$

where the auxiliary states $\left\{\rho_{i}(t)\right\}$ evolve as

$$
\begin{aligned}
& \frac{d \rho_{0}(t)}{d t}=-\gamma \rho_{0}(t), \\
& \frac{d \rho_{1}(t)}{d t}=-\gamma \rho_{1}(t)+\gamma \sigma_{z} \rho_{0}(t) \sigma_{z}, \\
& \frac{d \rho_{2}(t)}{d t}=+\gamma \sigma_{z} \rho_{1}(t) \sigma_{z},
\end{aligned}
$$

with initial conditions $\rho_{0}(0)=\rho_{0}$ and $\rho_{1}(0)=\rho_{2}(0)=0$. This Lindblad rate equation admits the stochastic representation defined previously. In fact, here the evolutions for the classical populations [Eq. [17)] are recovered from $P_{i}(t)=\operatorname{Tr}\left[\rho_{i}(t)\right], i=0,1,2$. On the other hand, the transformations $\rho \rightarrow \sigma_{z} \rho \sigma_{z}$ are taken into account in Eq. (22) through the coupling between the auxiliary states. The explicit solution for the system density matrix (21) recovers the result Eq. (21). 
Lindblad rate equations also arise from a generalized Born-Markov approximation. Hence, the previous dynamics [Eq. (22)] can alternatively be read as the result of the interaction with a complex structured reservoir [36].

\section{Bipartite Lindblad representation and measurement trajectories}

Lindblad rate equations can be embedded in a bipartite quantum dynamics [36]. In the present case, the "ancilla system" has three states, $|0\rangle,|1\rangle$, and $|2\rangle$. Denoting with $\rho_{t}^{s a}$ the system and ancilla density matrix, its evolution can be written as

$$
\begin{aligned}
\frac{d \rho_{t}^{s a}}{d t}= & +\frac{\gamma}{2}\left(\left[V_{1}, \rho_{t}^{s a} V_{1}^{\dagger}\right]+\left[V_{1} \rho_{t}^{s a}, V_{1}^{\dagger}\right]\right) \\
& +\frac{\gamma}{2}\left(\left[V_{2}, \rho_{t}^{s a} V_{2}^{\dagger}\right]+\left[V_{2} \rho_{t}^{s a}, V_{2}^{\dagger}\right]\right),
\end{aligned}
$$

where the initial state is $\rho_{0}^{s a}=\rho_{0} \otimes|0\rangle\langle 0|$. The dissipative channels are defined by the operators

$$
V_{1}=\sigma_{z} \otimes|1\rangle\left\langle 0\left|, \quad V_{2}=\sigma_{z} \otimes\right| 2\right\rangle\langle 1| .
$$

The dynamics given by the Lindblad rate equation (22) is recovered from $\rho_{i}(t)=\left\langle i\left|\rho_{t}^{s a}\right| i\right\rangle, i=0,1,2$.

The bipartite representation [Eq. (223)], through the standard quantum jump approach [1], allows to unravelling the evolution in measurement trajectories. Assuming that the measurement device detects the transitions $|0\rangle \rightarrow|1\rangle$ and $|1\rangle \rightarrow|2\rangle$, the bipartite measurement operators are $V_{1}$ and $V_{2}$. Similarly to Ref. [39], the "collisional trajectories," defined by Eq. (18), can be recovered from the bipartite measurement realizations. The main difference is that here only a finite number of transitions occur.

\section{Decoherence and recoherence}

The underlying quantum-classical dynamics can be generalized without affecting the non-Markovianity degree of the solution map. For example, the introduction of extra classical states also leads to the property $\lim _{t \rightarrow \infty} \rho_{t}=\rho_{0}$ if an even number of transformations (18) happen (Fig. 3). By increasing the number of collisions the phenomenon of recoherence occurs, that is, after an exponential decay the system coherence (almost) vanishes and emerges at a later time, recovering its initial value in the (stationary) long time regime.

Denoting the qubit coherence as $\rho_{12}(t)=c_{n}(t) \rho_{12}(0)$, where the function $c_{n}(t)$ gives its characteristic time behavior and the subindex $n$ denotes the (even) number of collisions, in Appendix $\mathrm{C}$ we obtain

$$
\begin{aligned}
c_{n}(t)= & e^{-2 \gamma t}+\int_{0}^{t} d t_{2} \int_{0}^{t_{2}} d t_{1} 2 \gamma e^{-2 \gamma\left(t_{2}-t_{1}\right)} \\
& \times\left[\frac{\gamma e^{-\gamma t_{1}}}{(n-1) !}\left(\gamma t_{1}\right)^{n-1}\right] .
\end{aligned}
$$

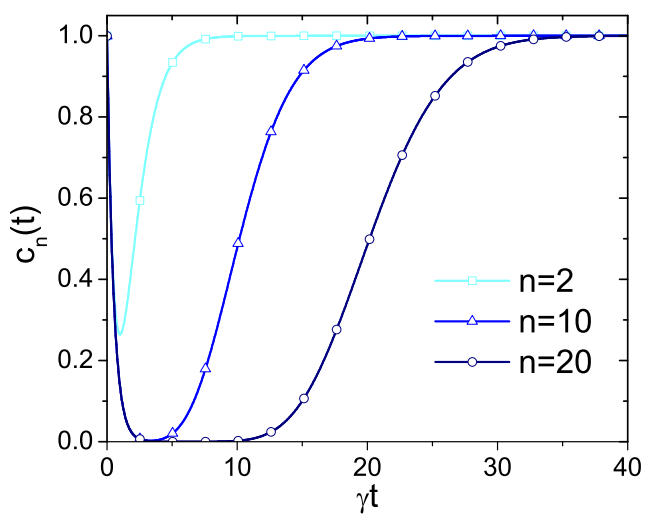

FIG. 4: Time dependence $c_{n}(t)$ of the qubit coherence for the extended dephasing model, Eq. (25), for different (even) number of collisional events, $n=2,10$, and 20 .

This expression follows by conditioning the quantum dynamics to a classical system with $n+1$ states, where each transition implies the transformation (18). The (unidirectional) coupling rate between successive states is $\gamma$. For the model with three states (Fig. 1), it follows $c_{2}(t)=1-2 \gamma t e^{-\gamma t}=e^{-\Gamma(t)}$, where consistently $\Gamma(t)$ is given by Eq. (5). In all cases the time dependent rate [Eq. (1)] follows as $\gamma(t)=(d / d t) \ln \left[1 / c_{n}(t)\right]$, with $\Gamma(t)=\ln \left[1 / c_{n}(t)\right]$, which fulfills Eq. (3).

In Fig. 4 it is plotted $c_{n}(t)$ as a function of time for $n=2,10$, and 20. For $n \gg 1$, the coherence vanishes following the decay behavior $c_{n}(t) \simeq \exp (-2 \gamma t)$, while recoherence always occurs in the long time regime, $\lim _{t \rightarrow \infty} c_{n}(t)=1$. On the other hand, a Markovian limit is recovered as $\lim _{n \rightarrow \infty} c_{n}(t)=\exp (-2 \gamma t)$. In this case, a standard collisional dynamics is recovered [38], which in a given realization contains an infinite number of disruptive transformations.

\section{B. Random unitary dynamics}

The Maximally non-Markovian random unitary dynamics defined by Eq. (9) and the probabilities (12) can also be derived taking into account an environment that is unaffected by the system dynamics. With this goal, in addition to the three auxiliary states defined by Eq. (22), an extra state $\rho_{M}(t)$ is introduced (Fig. 3). It is completely uncoupled of the other states and follows the Markovian evolution

$$
\frac{d \rho_{M}(t)}{d t}=\frac{1}{2} \gamma\left[\sigma_{x} \rho_{M}(t) \sigma_{x}-\rho_{M}(t)\right]
$$

Here, $\sigma_{x}$ is the $x$-Pauli matrix. The solution is given by

$$
\rho_{M}(t)=\frac{1}{2}\left[\left(1+e^{-\gamma t}\right) \rho_{0}+\left(1-e^{-\gamma t}\right) \sigma_{x} \rho_{0} \sigma_{x}\right] .
$$


The system density matrix is written as

$$
\rho_{t}=(1-r) \sum_{i=0}^{2} \rho_{i}(t)+r \rho_{M}(t),
$$

where $0 \leq r \leq 1$. This expression corresponds to a statistical mixture (or convex combination [43]) of a Markovian and a maximally non-Markovian dephasing evolution, Eqs. (26) and (11) respectively. Taking $r=1 / 2$, from the solutions (19) and (27) it follows the probabilities (12), recovering in consequence the maximally non-Markovian random unitary evolution presented in the previous section. Notice that also in this case the environment is unaware of the dependence of the system dynamics on its states, implying in consequence, the absence of any physical backflow of information. On the other hand, the state (28) can also be characterized in terms of Lindblad rate equations and bipartite evolutions.

It is interesting to note that Eq. (28) leads to a Markovian solution map for $r=1$. By an explicit calculation, it is possible to demonstrate that this is the unique value at which the map departs from a maximally nonMarkovian dynamics. In fact, for $0 \leq r<1$ we get $\gamma_{2}(t)+\gamma_{3}(t)=\gamma g_{a}(r, t) \nsupseteq 0, \lim _{t \rightarrow \infty} \Gamma_{1}(t)=\ln [1 /(1-r)]$, and $\lim _{t \rightarrow \infty} \Gamma_{2}(t)=\lim _{t \rightarrow \infty} \Gamma_{3}(t)=0$, where $g_{a}(r, t)=$ $[2(1-r)(1-\gamma t)] /\left[e^{\gamma t}-2(1-r) \gamma t\right]$. Hence, condition Eq. (11) is fulfilled. In contrast, a statistical mixture of a maximally entangled state and the identity matrix state (Werner state) departs from an entangled state at $r<1$ [44]. This difference is expectable because the non-Markovianity degree relies on a formal analogy with entanglement [13].

\section{NON-MARKOVIAN QUANTUM DYNAMICS WITHOUT ENVIRONMENT BACKFLOW OF INFORMATION}

The previous examples, as well as the results of Refs. [37, 39, 40], demonstrate that strong nonMarkovian effects may arise from simple quantumclassical hybrid dynamics. The environment consists in a set of classical degrees of freedom that follows their own dynamics. Here, we write the more general underlying equations that lead to these properties and analyze how memory effects may emerge.

\section{A. Discrete dissipative classical degrees of freedom}

The system density matrix is written in terms of a set of auxiliary states, $\rho_{t}=\sum_{R} \rho_{R}(t)$, whose evolution is given by a Lindblad rate equation,

$$
\begin{aligned}
\frac{d \rho_{R}(t)}{d t}= & \frac{-i}{\hbar}\left[H_{R}, \rho_{R}(t)\right]+\mathbb{L}_{R}\left[\rho_{R}(t)\right] \\
& -\sum_{R^{\prime}} \gamma_{R^{\prime} R} \rho_{R}(t)+\sum_{R^{\prime}} \gamma_{R R^{\prime}} \mathbb{E}_{R R^{\prime}}\left[\rho_{R^{\prime}}(t)\right],
\end{aligned}
$$

with initial conditions $\rho_{R}(0)=P_{R}(0) \rho_{0}$. In the first line, $H_{R}$ and $\mathbb{L}_{R}$ are the (conditional) unitary and dissipative Lindblad dynamics of the system given that the environment is in the state $R$. The second line introduces a coupling between the auxiliary states with rates $\left\{\gamma_{R^{\prime} R}\right\}$. In each transition $R^{\prime} \rightarrow R$, the state $\rho_{R^{\prime}}$ suffers the disruptive change $\mathbb{E}_{R R^{\prime}}\left[\rho_{R^{\prime}}\right] \rightarrow \rho_{R}$, where $\left\{\mathbb{E}_{R R^{\prime}}[\bullet]\right\}$ are trace preserving completely positive superoperators,

$$
\operatorname{Tr}\left(\mathbb{E}_{R R^{\prime}}[\rho]\right)=\operatorname{Tr}(\rho) .
$$

The probability that the environment is in the state $R$ at time $t$ is given by $P_{R}(t)=\operatorname{Tr}\left[\rho_{R}(t)\right]$ [36]. From Eq. (29), it follows the classical master equation

$$
\frac{d P_{R}(t)}{d t}=-\sum_{R^{\prime}} \gamma_{R^{\prime} R} P_{R}(t)+\sum_{R^{\prime}} \gamma_{R R^{\prime}} P_{R}^{\prime}(t) .
$$

This result implies that, in fact, the environment is completely unperturbed by the system state or dynamics. Hence, the environment is only a "spectator" or "casual bystander" during the whole evolution [40]. Eq. (29) is the more general quantum-classical evolution that leads to these properties [45].

The underlying equations (29) may induce strong nonMarkovian quantum effects, which in turn may depend on the initials conditions $\left\{P_{R}(0)\right\}$. For example, the particular case (22) is recovered with $H_{R} \rightarrow 0, \mathbb{L}_{R} \rightarrow 0$, and $\mathbb{E}_{R R^{\prime}}[\bullet] \rightarrow \sigma_{z} \bullet \sigma_{z}[46]$.

The possibility of observing memory or non-Markovian effects without a physical environment-to-system backflow of information follows from the unidirectional dependence of the system evolution on the environment states [Eq. [29)]. This dependence is present even when $\mathbb{E}_{R R^{\prime}}=\mathbb{I}$ or when $\left\{\gamma_{R^{\prime} R}\right\}=0$. In this last extreme case, $\left\{P_{R}(t)\right\}=\left\{P_{R}(0)\right\}$. By writing the system density matrix as $\rho_{t}=\Lambda_{t} \rho_{0}=V_{t, s} \Lambda_{t} \rho_{0}$, we realize that the intermediate propagator $V_{t, s}$ (which is used for defining the non-Markovianity degree [13]) may develops strong departures from a completely positive "Markovian" propagator. In fact, $V_{t, s}$ must to takes into account the previous dependence of the system dynamics on the environment states. This fact explain why, even when the environment is frozen $\left\{P_{R}(t)\right\}=\left\{P_{R}(0)\right\}$ [40], the system dynamics may develops memory effects without happening a physical environment-to-system backflow of information.

\section{B. Non-white Gaussian stochastic Hamiltonian}

Non-Markovian effects induced by non-white Gaussian stochastic Hamiltonians [35] also falls in the previous category. In order to show this fact, we write the density matrix as

$$
\rho_{t}=\int_{-\infty}^{+\infty} d \eta \rho_{\eta}(t)
$$


where the auxiliary state $\rho_{\eta}(t)$ depends on the real parameter $\eta$, and obeys the equation

$$
\begin{aligned}
\frac{\partial \rho_{\eta}(t)}{\partial t}= & \frac{-i}{\hbar}\left[H+\eta \Delta H, \rho_{\eta}(t)\right] \\
& -\gamma \frac{\partial\left[\eta \rho_{\eta}(t)\right]}{\partial \eta}+\frac{D}{2} \frac{\partial^{2} \rho_{\eta}(t)}{\partial \eta^{2}} .
\end{aligned}
$$

Both $H$ and $\Delta H$ are Hamiltonian operators. The environment dynamics, defined by the variable $\eta$, follows from $P_{\eta}(t) \equiv \operatorname{Tr}\left[\rho_{\eta}(t)\right]$, which gives

$$
\frac{\partial P_{\eta}(t)}{\partial t}=-\gamma \frac{\partial\left[\eta P_{\eta}(t)\right]}{\partial \eta}+\frac{D}{2} \frac{\partial^{2} P_{\eta}(t)}{\partial \eta^{2}} .
$$

This Fokker-Planck equation [47] is independent of the system state. Furthermore, the quantum state parametrically depends on the bath state $\eta$. Therefore, nonMarkovian effects obtained from Eq. (33) occur without a physical environment-to-system backflow of information. On the other hand, this equation can be mapped to a stochastic Hamiltonian evolution. In fact, Eq. (34) is equivalent to the Langevin equation [47]

$$
\frac{d}{d t} \eta(t)=-\gamma \eta(t)+\xi(t)
$$

where the white Gaussian noise $\xi(t)$ has correlation $\left\langle\xi(t) \xi\left(t^{\prime}\right)\right\rangle=D \delta\left(t-t^{\prime}\right)$. In contrast, the non-white Gaussian process $\eta(t)$ has an exponential correlation, which in the stationary regime reads $\left\langle\eta(t) \eta\left(t^{\prime}\right)\right\rangle_{\mathrm{st}}=$ $D /(2 \gamma) \exp \left(-\gamma\left|t-t^{\prime}\right|\right)$. In addition, the system density matrix $\rho_{t}$ [Eq. (32)] can be read as an average over realizations of $\eta(t), \rho_{t}=\left\langle\rho_{s t}(t)\right\rangle$, where $\rho_{s t}(t)$ follows the stochastic evolution

$$
\frac{d \rho_{s t}(t)}{d t}=\frac{-i}{\hbar}\left[H+\eta(t) \Delta H, \rho_{s t}(t)\right] .
$$

Therefore, stochastic non-white Hamiltonians also lead to non-Markovian effects [35] without happening a physical environment-to-system backflow of information.

\section{SUMMARY AND CONCLUSIONS}

Similarly to Schmidt number in entanglement theory, the degree of non-Markovianity allows to ranking memory effects developed by quantum evolutions. Maximally non-Markovian evolutions are the analog of maximally entangled states. Here, we showed that exist a class of such kind of extreme non-Markovian dynamics that can be modeled from simple quantum-classical hybrid dynamics. The environment, defined by the classical degrees of freedom, follows its own Markovian evolution, which in turn is unaffected by the system state or dynamics. Therefore, memory effects arise without the occurrence of any physical environment-to-system backflow of information. Departure with respect to a quantum Markovian regime follows from the unidirectional dependence of the system dynamics on the environment states.
Both dephasing and random unitary maximally nonMarkovian maps were modeled. In the former case, the dynamics can be read in terms of a finite (even) number of collisional events that change the sign of the qubit coherence, each event occurring at random times. The phenomena of decoherence and recoherence are captured in this frame. On the other hand, in the last case (random unitary maps), the system dynamics follows from a statistical mixture (convex combination) of the former nonMarkovian evolution and a Markovian dephasing channel in a different direction. In both cases, the classical degrees of freedom are unaffected by the system state. The underlying dynamics admit different representations such as in terms of quantum Markov chains, Lindblad rate equations, and measurement trajectories.

General Lindblad rate equations that lead to memory effects without a physical backflow of information were also characterized. The proposed models can be generalized in different ways (dimensionality, collision transformations, quantum ancillas, etc.), providing a basis for the study of a full family of maximally non-Markovian dynamics. This approach may be useful for modeling decoherence in quantum information channels where the environment is coupled during a set of finite intervals of time (collisional models with a finite number of events).

In accordance with previous results [37, 39, 40], the present analyses confirm that, in general, non-Markovian indicators or measures based solely on the system dynamics are unable to distinguish between memory (nonMarkovian) effects developed with or without a physical environment-to-system backflow of information.

\section{Acknowledgments}

This work was supported by Consejo Nacional de Investigaciones Científicas y Técnicas (CONICET), Argentina.

\section{Appendix A: Non-Markovianity degree}

Here, we briefly review the formalism of Ref. [13]. It is based on the divisibility of the density matrix propagator, $\rho_{t}=\Lambda_{t} \rho_{0}$. Splitting the time evolution as $\Lambda_{t}=$ $V_{t, s} \Lambda_{s}$, the solution map $\Lambda_{t}$ is called $k$-divisible if $V_{t, s}$ is $k$-positive for all $t \geq s \geq 0$, that is, $\mathbb{I}_{k} \otimes V_{t, s}$ is positive, where $\mathbb{I}_{k}$ is the identity operator in an extra Hilbert space of dimension $k$.

The degree of non-Markovianity is a number associated to a given evolution. The solution map $\Lambda_{t}$ has a nonMarkovianity degree $\operatorname{NMD}\left[\Lambda_{t}\right]=k$ if and only if $\Lambda_{t}$ is $(n-k)$ but not $(n+1-k)$ divisible. $\Lambda_{t}$ is Markovian if and only if $\operatorname{NMD}\left[\Lambda_{t}\right]=0$ and essentially non-Markovian if and only if $\operatorname{NMD}\left[\Lambda_{t}\right]=n$, where $n$ is the system Hilbert space dimension.

If $\Lambda_{t}$ is $k$-divisible, then $(d / d t)\left\|\mathbb{I}_{k} \otimes \Lambda_{t}\right\|_{1} \leq 0$, for all operators $X$ in the bipartite Hilbert space of the system 
and ancilla of dimension $k$. This result allows to defining a series of non-Markovianity measures. Departure from $k$ divisibility is measured with

$$
\mathcal{M}_{k}\left[\Lambda_{t}\right]=\sup _{X} \frac{N_{k}^{+}[X]}{\left|N_{k}^{-}[X]\right|}
$$

where

$$
N_{k}^{+}[X]=\int_{\lambda_{k}(X ; t)>0} \lambda_{k}(X ; t) d t,
$$

and, similarly for $N_{k}^{+}[X]$ (where the integral is over intervals such that $\left.\lambda_{k}(X ; t)<0\right)$, and

$$
\lambda_{k}(X ; t)=\frac{d}{d t}\left\|\mathbb{I}_{k} \otimes \Lambda_{t}\right\|_{1}
$$

The supremum is taken over all Hermitian $X$. Using this expression it follows $\int_{0}^{\infty} \lambda_{k}(X ; t) d t \leq 0$, which proves that $\left|N_{k}^{-}[X]\right| \geq N_{k}^{+}[X]$, and in consequence $\mathcal{M}_{k}\left[\Lambda_{t}\right] \in$ $[0,1]$. If $l>k$, then $\mathcal{M}_{l}\left[\Lambda_{t}\right] \geq \mathcal{M}_{k}\left[\Lambda_{t}\right]$ and, hence

$$
0 \leq \mathcal{M}_{1}\left[\Lambda_{t}\right] \leq \cdots \leq \mathcal{M}_{n}\left[\Lambda_{t}\right] \leq 1
$$

This expression is the analog of a similar relation between the coefficients of a Schmidt decomposition of an entangled pure quantum state. Maximally non-Markovian $d y$ namics corresponds to the case $\mathcal{M}_{1}\left[\Lambda_{t}\right]=1$, which immediately leads to

$$
\mathcal{M}_{1}\left[\Lambda_{t}\right]=\cdots=\mathcal{M}_{n}\left[\Lambda_{t}\right]=1 \text {, }
$$

in perfect analogy with maximally quantum entangled states [44].

The conditions under which the maps (1) and (8) are maximally non-Markovian [Eqs. (3) and (11) respectively] were derived in Ref. [13] from the condition $\mathcal{M}_{1}\left[\Lambda_{t}\right]=1$, and using, for example, the operator $X=\sigma_{x}$.

\section{Appendix B: Time-convoluted evolution for random unitary maps}

Given the evolution defined by the general random unitary map Eq. (9), the goal here is to obtain a timeconvoluted evolution

$$
\frac{d}{d t} \rho_{t}=\int_{0}^{t} d \tau \mathbb{L}_{t-\tau} \rho_{\tau},
$$

where $\mathbb{L}_{t}$ is a memory superoperator. The derivation is similar to the time-convolutionless case [41]. Expressing $\rho_{t}$ in terms of its propagator, $\rho_{t}=\Lambda_{t} \rho$, and working the previous time evolution in the Laplace domain $[f(z)=$ $\left.\int_{0}^{\infty} d t e^{-z t} f(t)\right]$, it follows the relation

$$
\mathbb{L}_{z}=\left(z \Lambda_{z}-1\right) \frac{1}{\Lambda_{z}}
$$

Writing Eq. (9) in the Laplace domain,

$$
\Lambda_{z} \rho=\sum_{\alpha=0}^{3} p_{\alpha}(z) \sigma_{\alpha} \rho \sigma_{\alpha}
$$

similarly to Ref. [41], let us observe that

$$
\Lambda_{z}\left(\sigma_{\alpha}\right)=\lambda_{\alpha}(z) \sigma_{\alpha},
$$

where the eigenvalues are given by

$$
\lambda_{\alpha}(z)=\sum_{\beta=0}^{3} H_{\alpha \beta} p_{\beta}(z),
$$

with $H_{\alpha \beta}$ being a square Hadamard matrix $H=$ $\{\{1,1,1,1\},\{1,1,-1,-1\},\{1,-1,1,-1\},\{1,-1,-1,1\}\}$ 41]. Notice that $\lambda_{0}(z)=1 / z$.

From Eqs. (B2) and (B4) it follows that

$$
\mathbb{L}_{z}\left(\sigma_{\alpha}\right)=\mu_{\alpha}(z) \sigma_{\alpha},
$$

where

$$
\mu_{\alpha}(z)=\frac{\left[z \lambda_{\alpha}(z)-1\right]}{\lambda_{\alpha}(z)} .
$$

Assuming that $\mathbb{L}_{z}$ can be written as

$$
\mathbb{L}_{z}(\rho)=\frac{1}{2} \sum_{\alpha=0}^{3} k_{\alpha}(z) \sigma_{\alpha} \rho \sigma_{\alpha},
$$

where $\left\{k_{\alpha}(z)\right\}$ are memory functions, using the property (B4) with $p_{\alpha}(z) \rightarrow k_{\alpha}(z)$, it follows that $\mathbb{L}_{z}\left(\sigma_{\alpha}\right)=(1 / 2) \sum_{\alpha=0}^{3} H_{\alpha \beta} k_{\beta}(z) \sigma_{\alpha}$. From this relation and Eq. (B6), and by using that $H^{-1}=(1 / 4) H$, it follows a close expression for the memory functions

$$
k_{\alpha}(z)=\frac{1}{2} \sum_{\beta=0}^{3} H_{\alpha \beta} \mu_{\beta}(z) .
$$

Finally, by using that the sum $\sum_{\beta=0}^{3} k_{\alpha}(z)=$ $(1 / 2) \sum_{\beta=0}^{3} H_{\alpha \beta} \mu_{\beta}(z)=\mu_{0}(z)=0$, if follows the final standard form

$$
\mathbb{L}_{z}(\rho)=\frac{1}{2} \sum_{j=1}^{3} k_{j}(z)\left(\sigma_{j} \rho \sigma_{j}-\rho\right) .
$$

The memory functions $\left\{k_{\alpha}(z)\right\}$ only depend on the set of probabilities $\left\{p_{\alpha}(z)\right\}$. On the other hand, general kernels $\left\{k_{\alpha}(z)\right\}$ that guarantee a completely positive solution map were characterized in Ref. [42].

For the dephasing map defined by Eqs. (11) and (4), the probabilities are $p_{0}(t)=1-\gamma t \exp (-\gamma t), p_{1}(t)=p_{2}(t)=$ 0 , and $p_{3}(t)=\gamma t \exp (-\gamma t)$, Eq. (20). From Eq. (B9) it follows $k_{1}(z)=k_{2}(z)=0$, and $k_{3}(z)=2 z^{2} \gamma /\left(z^{2}+\gamma^{2}\right)$, which consistently is the Laplace transform of Eq. (77). For the probabilities Eq. (12), the kernels have the same structure, where their specific time dependence can be written in terms of exponential and trigonometric functions. 


\section{Appendix C: Decoherence and recoherence calculus}

Here, we derive the coherence behavior for a collisional dynamics where $n$-applications of the disruptive transformation $\rho \rightarrow \sigma_{z} \rho \sigma_{z}$ are applied at random times. The probability density (waiting function) for the consecutive intervals is $w_{t}=\gamma \exp (-\gamma t)$, which has associated the survival probability $s_{t}=1-\int_{0}^{t} w_{t^{\prime}} d t^{\prime}=\exp (-\gamma t)$. The following derivation is valid for any $w_{t}$.

The coherence is written as $\rho_{12}(t)=c_{n}(t) \rho_{12}(0)$. Given that an even finite number of collisions $(n)$ happen, that each collision changes the sign of the coherence, in the Laplace domain $\left[f(z)=\int_{0}^{\infty} d t e^{-z t} f(t)\right]$ it follows

$$
\begin{aligned}
c_{n}(z)= & s_{z}\left(1+w_{z}^{2}+w_{z}^{4}+\cdots w_{z}^{n-2}\right)+\frac{w_{z}^{n}}{z} \\
& -s_{z}\left(w_{z}+w_{z}^{3}+\cdots w_{z}^{n-1}\right)
\end{aligned}
$$

where even and odd numbers of intermediate collisions were taken into account. Rewriting the previous expression as

$$
c_{n}(z)=s_{z}\left(1-w_{z}\right) \sum_{i=0}^{\frac{n-2}{2}}\left(w_{z}^{2}\right)^{i}+\frac{w_{z}^{n}}{z}
$$

using that $\sum_{i=0}^{m} a^{i}=\frac{1-a^{m+1}}{1-a}$, and that $s_{z}=\left[1-w_{z}\right] / z$, it follows

$$
c_{n}(z)=\left(\frac{1-w_{z}}{z}\right) \frac{\left(1-w_{z}^{n}\right)}{\left(1+w_{z}\right)}+\frac{w_{z}^{n}}{z} .
$$

The previous expression is valid for arbitrary $w_{z}$. In the proposed dynamics, $w_{z}=\gamma /(z+\gamma)$, leading to

$$
c_{n}(z)=\frac{1}{z+2 \gamma}+\frac{1}{z}\left(\frac{2 \gamma}{z+2 \gamma}\right)\left(\frac{\gamma}{z+\gamma}\right)^{n} .
$$

In the time domain this expression recovers Eq. (25). From $\lim _{z \rightarrow 0} z c_{n}(z)=1$, it follows $\lim _{t \rightarrow \infty} c_{n}(t)=1$. On the other hand, a Markovian limit is recovered as $\lim _{n \rightarrow \infty} c_{n}(z)=1 /(z+2 \gamma)$.
[1] H. P. Breuer and F. Petruccione, Theory of Open Quantum Systems (Oxford University Press, Oxford, England, 2002).

[2] I. de Vega and D. Alonso, Dynamics of non-Markovian open quantum systems, Rev. Mod. Phys. 89, 015001 (2017).

[3] R. Alicki and K. Lendi, Quantum Dynamical Semigroups and Applications, Lect. Notes Phys. 717 (Springer, Berlin Heidelberg, 2007).

[4] H. P. Breuer, E. M. Laine, J. Piilo, and V. Vacchini, Colloquium: Non-Markovian dynamics in open quantum systems, Rev. Mod. Phys. 88, 021002 (2016); H. P. Breuer, Foundations and measures of quantum nonMarkovianity, J. Phys. B 45, 154001 (2012).

[5] A. Rivas, S. F. Huelga, and M. B. Plenio, Quantum non-Markovianity: characterization, quantification and detection, Rep. Prog. Phys. 77, 094001 (2014).

[6] H. P. Breuer, E. M. Laine, and J. Piilo, Measure for the Degree of Non-Markovian Behavior of Quantum Processes in Open Systems, Phys. Rev. Lett. 103, 210401 (2009); E. M. Laine, J. Piilo, and H. P. Breuer, Measure for the non-Markovianity of quantum processes, Phys. Rev. A 81, 062115 (2010).

[7] M. M. Wolf, J. Eisert, T. S. Cubitt, and J. I. Cirac, Assessing Non-Markovian Quantum Dynamics, Phys. Rev. Lett. 101, 150402 (2008); M. M. Wolf and J. Ignacio Cirac, Dividing Quantum Channels, Commun. Math. Phys. 279, 147 (2008).

[8] A. Rivas, S. F. Huelga, and M. B. Plenio, Entanglement and Non-Markovianity of Quantum Evolutions, Phys. Rev. Lett. 105, 050403 (2010).

[9] S. C. Hou, S. L. Liang, and X. X. Yi, Non-Markovianity and memory effects in quantum open systems, Phys. Rev.
A 91, 012109 (2015)

[10] J. Bae and D. Chruściński, Operational Characterization of Divisibility of Dynamical Maps, Phys. Rev. Lett. 117, 050403 (2016).

[11] S. Lorenzo, F. Plastina, and M. Paternostro, Geometrical characterization of non-Markovianity, Phys. Rev. A 88, 020102(R) (2013).

[12] M. J. W. Hall, J. D. Cresser, L. Li, and E. Andersson, Canonical form of master equations and characterization of non-Markovianity, Phys. Rev. A 89, 042120 (2014); J. D. Cresser and C. Facer, Master equations with memory for systems driven by classical noise. Opt. Commun. 283, 773 (2010).

[13] D. Chruściński and S. Maniscalco, Degree of NonMarkovianity of Quantum Evolution, Phys. Rev. Lett. 112, 120404 (2014).

[14] N. K. Bernardes, A. Cuevas, A. Orieux, C. H. Monken, P. Mataloni, F. Sciarrino, and M. F. Santos, Experimental observation of weak non-Markovianity, Sci. Rep. 5, 17520 (2015).

[15] D. Chruściński, C. Macchiavello, and S. Maniscalco, Detecting Non-Markovianity of Quantum Evolution via Spectra of Dynamical Maps, Phys. Rev. Lett. 118, 080404 (2017).

[16] Md. M. Ali, P. -Y. Lo, M. W. -Y. Tu, and W. -M. Zhang, Non-Markovianity measure using two-time correlation functions, Phys. Rev. A 92, 062306 (2015); G. Guarnieri, A. Smirne, and B. Vacchini, Quantum regression theorem and non-Markovianity of quantum dynamics, Phys. Rev. A 90, 022110 (2014); A. A. Budini, Operator Correlations and Quantum Regression Theorem in Non-Markovian Lindblad Rate Equations, J. Stat Phys. 131, 51 (2008). 
[17] K. Luoma, P. Haikka, and J. Piilo, Detecting nonMarkovianity from continuous monitoring, Phys. Rev. A 90, 054101 (2014).

[18] X.-M. Lu, X. Wang, and C. P. Sun, Quantum Fisher information flow and non-Markovian processes of open systems, Phys. Rev. A 82, 042103 (2010).

[19] S. Luo, S. Fu, and H. Song, Quantifying nonMarkovianity via correlations, Phys. Rev. A 86, 044101 (2012).

[20] A. K. Rajagopal, A. R. Usha Devi, and R. W. Rendell, Kraus representation of quantum evolution and fidelity as manifestations of Markovian and non-Markovian forms, Phys. Rev. A 82, 042107 (2010).

[21] F. F. Fanchini, G. Karpat, B. Çakmak, L. K. Castelano, G. H. Aguilar, O. Jiménez Farías, S. P. Walborn, P. H. Souto Ribeiro, and M. C. de Oliveira, Non-Markovianity through Accessible Information, Phys. Rev. Lett. 112, 210402 (2014).

[22] B. Vacchini, A. Smirne, E. -M. Laine, J. Piilo, and H. P. Breuer, Markovianity and non-Markovianity in quantum and classical systems, New Journal of Physics 13, 093004 (2011).

[23] S. Wißmann, H. P. Breuer, and B. Vacchini, Generalized trace-distance measure connecting quantum and classical non-Markovianity, Phys. Rev. A 92, 042108 (2015).

[24] D. Chruściński, A. Kossakowski, and A. Rivas, Measures of non-Markovianity: Divisibility versus backflow of information, Phys. Rev. A 83, 052128 (2011).

[25] P. Haikka, J. D. Cresser, and S. Maniscalco, Comparing different non-Markovianity measures in a driven qubit system, Phys. Rev. A 83, 012112 (2011).

[26] M. Jiang and S. Luo, Comparing quantum Markovianities: Distinguishability versus correlations, Phys. Rev. A 88, 034101 (2013).

[27] C. Addis, B. Bylicka, D. Chruściński, and S. Maniscalco, Comparative study of non-Markovianity measures in exactly solvable one- and two-qubit models, Phys. Rev. A 90, 052103 (2014).

[28] F. Benatti, D. Chruściński, and S. Filippov, Tensor power of dynamical maps and positive versus completely positive divisibility, Phys. Rev. A 95, 012112 (2017).

[29] B. Bylicka, M. Johansson, and A. Acín, Constructive Method for Detecting the Information Backflow of NonMarkovian Dynamics, Phys. Rev. Lett. 118, 120501 (2017).

[30] F. A. Wudarski and F. Petruccione, Exchange of information between system and environment: Facts and myths, Euro Phys. Lett. 113, 50001 (2016).

[31] G. Guarnieri, C. Uchiyama, and B. Vacchini, Energy backflow and non-Markovian dynamics, Phys. Rev. A 93, 012118 (2016).

[32] G. Guarnieri, J. Nokkala, R. Schmidt, S. Maniscalco, and B. Vacchini, Energy backflow in strongly coupled non-Markovian continuous-variable systems, Phys. Rev. A 94, 062101 (2016).

[33] R. Schmidt, S. Maniscalco, and T. Ala-Nissila, Heat flux and information backflow in cold environments, Phys. Rev. A 94, 010101(R) (2016).

[34] M. Wittemer, G. Clos, H. P. Breuer, U. Warring, and T. Schaetz, Measurement of quantum memory effects and its fundamental limitations, Phys. Rev. A 97, 020102(R) (2018).

[35] A. A. Budini, Quantum systems subject to the action of classical stochastic fields, Phys. Rev. A 64, 052110 (2001); T. Grotz, L. Heaney, and W. T. Strunz, Quantum dynamics in fluctuating traps: Master equation, decoherence, and heating, Phys. Rev. A 74, 022102 (2006); J. Trapani, M. Bina, S. Maniscalco, and M. G. A. Paris, Collapse and revival of quantum coherence for a harmonic oscillator interacting with a classical fluctuating environment, Phys. Rev. A 91, 022113 (2015); J. I. Costa-Filho, R. B. B. Lima, R. R. Paiva, P. M. Soares, W. A. M. Morgado, R. Lo Franco, and D. O. Soares-Pinto, Enabling quantum non-Markovian dynamics by injection of classical colored noise, Phys. Rev. A 95, 052126 (2017).

[36] A. A. Budini, Lindblad rate equations, Phys. Rev. A 74, 053815 (2006).

[37] A. A. Budini, Post-Markovian quantum master equations from classical environment fluctuations, Phys. Rev. E 89, 012147 (2014).

[38] A. A. Budini, Stochastic representation of a class of nonMarkovian completely positive evolutions, Phys. Rev. A 69, 042107 (2004); A. A. Budini and P. Grigolini, Non-Markovian nonstationary completely positive openquantum-system dynamics, Phys. Rev. A 80, 022103 (2009); B. Vacchini, Non-Markovian master equations from piecewise dynamics, Phys. Rev. A 87, 030101(R) (2013).

[39] A. A. Budini, Embedding non-Markovian quantum collisional models into bipartite Markovian dynamics, Phys. Rev. A 88, 032115 (2013).

[40] N. Megier, D. Chruściński, J. Piilo, and W. T. Strunz, Eternal non-Markovianity: from random unitary to Markov chain realisations, Sci. Rep. 7, 6379 (2017).

[41] D. Chruściński and F. A. Wudarski, Non-Markovian random unitary qubit dynamics, Phys. Lett. A 377, 1425 (2013); D. Chruściński and F. A. Wudarski, NonMarkovianity degree for random unitary evolution, Phys. Rev. A 91, 012104 (2015).

[42] F. A. Wudarski, P. Nalezyty, G. Sarbicki, and D. Chruściński, Admissible memory kernels for random unitary qubit evolution, Phys. Rev. A 91, 042105 (2015); K. Siudzińska and D. Chruściński, Memory kernel approach to generalized Pauli channels: Markovian, semi-Markov, and beyond, Phys. Rev. A 96, 022129 (2017).

[43] F. A. Wudarski and D. Chruściński, Markovian semigroup from non-Markovian evolutions, Phys. Rev. A 93, 042120 (2016).

[44] R. Horodecki, P. Horodecki, M. Horodecki, and K. Horodecki, Quantum entanglement, Rev. Mod. Phys. 81, 865 (2009).

[45] The demonstration can straightforwardly be performed by taking an arbitrary Lindblad rate equation [36] for a set of auxiliary states $\left\{\rho_{R}(t)\right\}$ and demanding that $\operatorname{Tr}\left[\rho_{R}(t)\right]=P_{R}(t) \forall R$, where the set of probabilities $\left\{P_{R}(t)\right\}$ obey the classical master equation (31).

[46] Taking arbitrary initial conditions in Eq. (22), $\rho_{k}(0)=$ $\rho_{0} q_{k}$, with $\sum_{k=0}^{2} q_{k}=1$, it follows $\rho_{0}(t)=q_{0} e^{-\gamma t} \rho_{0}$, $\rho_{1}(t)=q_{1} e^{-\gamma t} \rho_{0}+q_{0} \gamma t e^{-\gamma t} \sigma_{z} \rho_{0} \sigma_{z}$, and $\rho_{2}(t)=\left\{q_{2}+\right.$ $\left.q_{0}\left[1-e^{-\gamma t}(1+\gamma t)\right]\right\} \rho_{0}+q_{1}\left(1-e^{-\gamma t}\right) \sigma_{z} \rho_{0} \sigma_{z} \rho_{0}$. From Eq. (10), the rate $\gamma(t)$ in Eq. (11) becomes $\gamma(t)=$ $2 \gamma\left[q_{0}(1-\gamma t)+q_{1}\right] /\left[e^{\gamma t}\left(q_{0}-q_{1}+q_{2}\right)-2 q_{0} \gamma t+2 q_{1}\right]$, while $\lim _{t \rightarrow \infty} \int_{0}^{t} \gamma\left(t^{\prime}\right) d t^{\prime}=\ln \left[1 /\left(q_{0}-q_{1}+q_{2}\right)\right]$.

[47] N. G. van Kampen, Stochastic Processes in Physics and Chemistry, (North-Holland, Amsterdam, 1992). 